\title{
A risk-based tool for documenting and auditing the modelling process
}

\author{
$\underline{\text { J.H.A. Guillaume }}^{\mathrm{a}}$ \\ ${ }^{a}$ Integrated Catchment Assessment and Management (iCAM) Centre, The Fenner School of Environment and \\ Society, The Australian National University, Australian Capital Territory \\ Email: joseph.guillaume@anu.edu.au
}

\begin{abstract}
Integrated modelling literature recognises the need to focus on process in model development, and provide documentation to allow critical review. It should be possible to critique key judgement calls made by the modeller. In order to achieve this, the whole modelling process needs to be documented in sufficient detail that ideally it could be replicated. Existing documentation, in reports and journals, rarely achieves the required level of detail in describing the modelling process, as it represents the authors' necessarily restricted view of the audience's immediate needs. While methods may vary between projects, the same uncertainties are usually encountered along a broadly similar set of model development steps. This paper therefore proposes an approach, and a supporting tool, to systematically document uncertainties rather than tasks. An uncertainty is a reason for doubting that the right choice was made. Uncertainties arise from boundary judgements, i.e. in choosing one course of action and excluding alternatives, there may be a reason that the right or 'true' value may be missed. To manage uncertainty, a set of uncertainty management actions need to be addressed. To identify whether an uncertainty has been satisfactorily managed, the impact of the uncertainty on the end user's objectives, i.e. the corresponding risk, must be acceptable. A hierarchical documentation format is used to provide a consistent but extensible structure, minimising input required and maximising flexibility in manipulating the data to provide output reports. As an audit tool, this approach and tool can be used to evaluate the completeness of documentation, and allow step-by-step review of a modelling process. It can also be used by the modeller as an aid to reflection on the model development process to ensure uncertainty is treated thoroughly. By building a shared catalogue of workflow elements for modelling and uncertainty management, the documentation structure can: alert the modeller to missed uncertainties; inform the development of best practice guidelines; and allow consistent communication of accumulated uncertainties and their implications for even a large multi-disciplinary geographicallydistributed integrated modelling project. The approach and supporting tool is compared with a targeted literature review highlighting the roles in quality assurance documentation of guidelines, tasks vs. boundary judgements and uncertainty. Issues are discussed related to the work involved in detailed documentation, use of the large amount of information collected and adoption of such tools. The argument is made that appropriately designed information technology software (in prototype development) is able to minimise these problems, and help the documentation approach deliver its multiple benefits to both individual modellers and the larger modelling, uncertainty and risk management community.
\end{abstract}

Keywords: Documentation, uncertainty, risk, quality assurance 


\section{INTRODUCTION}

This paper presents an approach to documentation to facilitate quality assurance. It is recognised that a predictive model, particularly when used to predict future conditions, cannot be validated by comparison to historical data (Refsgaard and Henriksen, 2004, Wood and Mason, 1979). Quality assurance of the model development process is therefore required to complement the validation of a model using observed data (Jakeman et al., 2006). To facilitate this task, the model and its development process must be adequately documented to allow critical review. This paper presents an approach to documentation based on describing the treatment of uncertainties, rather than tasks. It also outlines the characteristics of a prototype software tool to support the approach. Section 1 introduces key terms, aims and uses cases for this documentation approach. Section 2 briefly summarises the documentation content requirements, and software and format capabilities. Section 3 compares notable theoretical and practical features of this approach with existing documentation approaches. Section 4 discusses the practical issues involved in the use of the approach and tool. This paper focuses on presenting the properties of the documentation approach and tool, with earlier papers presenting more detail on this view of uncertainty (Guillaume et al., 2010) and applications of the tool (Guillaume et al., 2011, Guillaume and Pierce, 2011).

\subsection{Definitions and Aims}

Quality assurance aims to "assure technically and scientifically adequate execution of all tasks included in the study, and to assure that all modelling-based analysis is reproducible and defensible" (Refsgaard et al., 2005). From the point of view of uncertainty, this is equivalent to assuring that after they are managed, the uncertainties arising from boundary judgements have an acceptable impact on the objectives of the modelling-based analysis. This view of quality assurance is fundamental to this paper, so its key terms will now be defined. In this context, the process of assuring involves using documentation to expose existing subjective judgements to allow outside critique. A boundary judgement is a choice that excludes alternatives, for example the choice of model parameter values. An uncertainty is a reason for doubting that the right choice was made, e.g. error in calibration data may be reason to believe that the 'true' model parameters have been excluded, or 'false' model parameters were chosen. Managing uncertainty involves seven actions (Guillaume et al., 2010): identifying, prioritising, reducing, describing, propagating and communicating reasons for doubt, as well as anticipating the future treatment of residual and unrecognised reasons for doubt. The concept of uncertainty impacting on objectives corresponds to the definition of risk (ISO, 2009). Obtaining an acceptable impact therefore corresponds to minimising the risk that the analysis was not executed adequately, technically or scientifically, or that it was not reproducible or defensible.

The approach and tool described therefore aim to provide documentation to allow the modeller and a potential assessor to review the treatment of uncertainty throughout the model and its development process. It should be possible to critique each judgement call made by the modeller. The tool is not intended for enduser documentation, though some of its outputs may be useful for this purpose. The documentation approach also aims to:

- Be systematic, covering the process and its uncertainties as comprehensively as possible. This requires enforcing a certain structure to the documentation. All steps in the process should be able to be recovered from documentation, but they need not be replicable (Checkland and Holwell, 2007). From the point of view of quality assurance, if the recovered process were replicated, it would be possible for a different researcher to make different decisions (Refsgaard et al., 2006), but still accept the rigor of the original process.

- Expose currently implicit judgements in the modelling process. This approach recognises the importance of informal knowledge through the role of intuition and experience in the craft of modelling (Jakeman et al., 2006). The documentation therefore emphasises the role of making sense of the process rather than the often conflicting aims of planning (other than by allow review of plans), and providing guidelines (though a documented process could be used to inform best practice).

- Be transferable, and widely usable. While it is being tested in a groundwater management modelling context, it is built from high level principles intended to be useful in a variety of decision support modelling situations. As a result, the only assumptions made about model development are that it consists of a separable sequence of boundary judgements with an objective. 


\subsection{Use cases}

Within its aims, the documentation tool may be used in a variety of ways, depending on the need for thoroughness, sharing knowledge and reflection on the process. Uses of the tool can evolve, becoming more sophisticated as required. A project manager might start by choosing the approach to quickly document what was done. The tool might then be used to review more advanced methods available as they are needed. As the first phase of the project finishes, the steps documented are augmented with consideration of the uncertainties faced and how they were addressed. This is used by external reviewers to assess the study. In parallel, the same process is undertaken for other past projects, and the results are used to demonstrate the quality of work by the research group even where project management is not based on explicit documentation and review. Some areas for improvement are identified, and the next phase of the project chooses to use the documentation tool to ensure uncertainties are explicitly addressed. At each stage of the project, the measures planned are documented, and their success reviewed. It is noticed that the documentation accumulated can contribute to other planned projects, so it is integrated into the shared knowledge base, with minimal changes required thanks to the software platform. The final phase of the project involves high stakes and collaboration between several groups in a large multi-disciplinary geographically-distributed integrated modelling project. The documentation approach provides a common structure for communication, and enables everybody to be aware of the uncertainties present. The adaptive project management ensures that at each stage of the project, all parties are comfortable that their requirements are met, and that the knowledge they contribute has been taken into account. The software tool is not yet ready for such sophisticated use, but has been used to structure a groundwater management literature review (Guillaume and Pierce, 2011) and to outline the treatment of uncertainty in a hypothetical modelling situation (Guillaume et al., 2011).

\section{IMPLEMENTATION DESCRIPTION}

This section will present the content requirements of the documentation and technical details of the software tool and file format. The format deliberately allows sufficient flexibility to allow the process of using the documentation to vary depending on context, as suggested in the use cases. It is however intended that the tool will be most useful in the context of iterative evaluation of progress during a project. The tool would be used for every step - after initial planning, and revisited after the step is complete, and each time results from that step are used. It is also intended that external guidelines or documentation from literature reviews and previous model assessments would be integrated and used during the documentation of a new project.

\subsection{Documentation content}

The content of the documentation and its hierarchical structure is shown in Figure 1. The basic content is a set of steps in the process of model development and use, which have associated uncertainties. These uncertainties in turn have a set of uncertainty management actions, the treatment of which needs to be documented. In addition, each step is associated with an aim. Each uncertainty is associated with its classification by 'nature' and 'level' (Guillaume et al., 2010), an assessment of its possible effects on objectives, and the residual risk from the steps taken to address the uncertainty. The format is by design extensible, and allows other content to be embedded. In particular, justifications for choice of treatments of uncertainty are encouraged, e.g. by citing references. Extendibility has already been used in one case to allow documentation to be extracted by citation and by date.

The steps are intentionally undefined here. They are intended to be defined by the modeller to fit their conceptualisation of the process. This may be the steps dictated by an accepted guideline, but does not need to be. The aims of each step are identified. To help identify the uncertainties, it is recommended that the steps be hierarchically decomposed into a set of atomic 'boundary judgements' (Midgley, 2000). Boundary judgements are steps where a choice was made to use some method, parameter, and therefore the complementary choice was (implicitly) made not to use other available methods or parameters. At the level of boundary judgement, the aims will generally imply obvious uncertainties that may prevent the aim from being satisfied. This documentation approach does not strictly require that this method of identifying uncertainties be used. This method is intended as a thorough method of identifying uncertainties as completely as possible. Depending on QA requirements, such a high level of confidence may not be required, in which case uncertainties may be associated with a step representing an aggregation of boundary judgements.

For each uncertainty, the user is required to document how each uncertainty management action will be addressed (Guillaume et al., 2010). These actions are: how resources to address uncertainty are prioritised, how the uncertainty will be reduced, described, propagated to obtain uncertainty in objectives, 
communicated to decision makers or end users. There will commonly be residual uncertainty and unrecognised uncertainties. Measures to anticipate and manage them also need to be documented.

Determining quality assurance acceptability criteria is a social, not just a scientific issue (Refsgaard and Henriksen, 2004). In order to ensure decision makers are satisfied with the treatment of uncertainty, it needs to be placed into its context, in terms of its possible consequences, i.e. risk. The documentation therefore requires that the consequences of the uncertainty (i.e. risk), and the consequences from the residual uncertainty be documented. If a model is re-used, aims and risks may need to be modified. While aims and risks can be revised, and may result in a process being judged inadequate, the rigour of the process should always be judged with respect to its stated aims and risks.

- High level step 1

○ Aims

○ Boundary judgment 1

- Aims

- Uncertainty 1

- Classification (Nature and Level)

- Potential risk: consequences on objectives

- How it will be:

o Prioritised

○ Reduced

- Described

- Propagated

○ Communicated

- Potential residual risk

- How residual uncertainty (and consequences) will be anticipated and managed

- Uncertainty $2 \ldots$

○ Boundary judgment $2 \ldots$

- High level step 2 ...

Figure 1 Hierarchical structure of documentation content

\subsection{Documentation format and software}

The documentation format and software is currently a prototype. It includes a proof of concept for input of documentation and output of reports, both interactively and offline, as well as for integration of documentation from multiple projects into a shared knowledge base. The documentation format is based on $\mathrm{xml}$, chosen as a standard format with well-developed tools. The Python lxml library (lxml developers, 2011) provides the key capabilities - parsing, modification of the DOM tree, search and selection/extraction (XPATH).

For input, four methods have been developed, tailored to particular use cases. Spot changes can be made by converting to a text format and using a text editor, mind-mapping or outlining software. Spot additions to an existing tree, e.g. for a literature review, is assisted by a series of GUI dialog windows. A series of dialogs also enable the identification of model steps, and given those steps, guide the user through editing the rest of the content. These dialogs auto-complete the names of uncertainties and steps based on existing entries to help ensure consistency.

The software allows subsets of documentation to be extracted in various forms related to specific needs by selecting tags, nodes and sub-trees by tag and text content. The key reports include: summarising steps followed, uncertainties for all steps and their treatment, summary of consequences of all uncertainties, collected residual risk, and all treatments recommended for particular uncertainty management actions, e.g. propagation of uncertainty, communicating uncertainty, and anticipating and managing residual uncertainty. There are also capabilities for specific requirements, such as for custom tags for dates, references, and model inputs and outputs. These outputs are represented as plain text, html tables, mind-maps, radial diagrams and as interactive presentations.

A number of capabilities do not exist but are regarded as important. The ability to combine documents into a knowledge base is currently limited. The software can identify orthographically similar terms corresponding to same concept and merge trees with duplicated uncertainties and steps. Interactive dialogs would guide the quality assurance process by iterating through uncertainties to allow assessment of their treatment. Changes 
in a single document would be merged into a shared database, making use of a thesaurus of terminology, effectively creating an evolving, self-generated ontology. This would be supplemented with literature reviews, and existing best practice guidelines.

\section{COMPARISON WITH LITERATURE}

Model documentation has been discussed since the early days of punch-cards (Gass et al., 1981). It is recognised as a non-trivial problem, and the literature has extensively identified issues, and quality requirements. This discussion will focus on comparison of key theoretical features and practical considerations with prior work with a common aim of quality assurance (Refsgaard et al., 2005, Scholten and Udink ten Cate, 1999). The literature also recognises the need to tailor documentation to purpose and audience (Refsgaard et al., 2005). This tool is restricted to the purpose of providing a "written record for communication and quality control" (Highland, 1977) by modellers and assessors.

This tool has taken a fundamentally different approach to other documented quality assurance approaches by avoiding setting best practice guidelines (Refsgaard and Henriksen, 2004, Scholten et al., 2001, Refsgaard et al., 2006). Instead, the tool facilitates the review of the full process against individual judgement. Guidelines imply an objective benchmark of normative 'quality', particularly where scoreboards are used. It is recognised that the appropriateness of guidelines depends on the maturity of science and the market for consulting model studies (Scholten and Udink ten Cate, 1999, Refsgaard et al., 2005), e.g. with hydrological modelling in the Netherlands (Scholten et al., 2001). In a sense, this tool fills a niche, emphasises the subjective and constructive dialogue side of quality assurance. Both approaches are "forcing modellers to work in a structured manner, without being forced into a straitjacket by strictly prescribing certain methods" (Scholten et al., 2001). Providing guidelines is however too great a straitjacket in some circumstances. There are also other examples of this alternative approach - recording modelling actions, products, and choices but not making prescriptions (Scholten and Udink ten Cate 1999).

The tool requires the user to specify steps, hierarchically refined to the level of boundary judgements, rather than defining tasks to document (Refsgaard et al., 2006, Brade, 2000, Wood, 1986). The fundamental building block - boundary judgements are a central feature of process philosophy as formulated by Midgley (2000). By making the "process of bringing knowledge into being" analytically prime, it allows the object/subject dichotomy to be overcome which enables identical critique of both the decision and the way in which it was made. This philosophical approach also relates uncertainty analysis and quality assurance to the simpler concept of critiquing boundary judgements (Midgley, 2000). The process of defining the steps is key to the quality assurance process by enabling the modeller to make sense of what has been done in order to then critique it. In practice, the highest level steps have been pre-defined as a decision pathway (Guillaume and Pierce, 2011), forming a common base for a shared hierarchy, but sub-steps were specified for each project separately.

This approach retains other advantages of using a pre-defined set of tasks for documentation. It allows detailed, more thorough quality assessment (Wood and Mason, 1979, Scholten and Udink ten Cate, 1999), and planning and scheduling of progress reviews (Wood and Mason, 1979). Guidelines can also help by providing clarity about tasks (Henriksen et al., 2009), as long as they have been defined by consensus with a number of stakeholders (Scholten et al., 2001). This has deliberately been forgone given the difficulties in situations where such a consensus cannot be reached. As an "intrinsically continuous and overlapping process" (Wood and Mason, 1979), tasks are arbitrary (Henriksen et al., 2009) and are not universal due to different mental models of the process (Scholten and Udink ten Cate 1999). Our experience has shown that it is useful to at least allow tasks to be reordered. The MoST tool (Refsgaard et al., 2006) does provide tools to edit the tasks in a knowledge base. Such a database is a valuable resource, but there is still a fundamental difference between editing guidelines and allowing the editing of a knowledge base in the documentation process itself. To return to the question of clarity of tasks, the same aim can be achieved with this tool through diagnostic questions to ensure that the boundary judgements cannot be further sub-divided and the uncertainties are uniquely classifiable (Warmink et al., 2010). In agreement with the literature, it is crucial for a shared knowledge base to have a consistent terminology (Refsgaard and Henriksen, 2004), which we argue should take the form of a thesaurus integrated into the documentation software, to relate 'official' phrases to other commonly used expressions.

The key element of this documentation approach is uncertainty, not tasks. Goals can vary, but problems are often similar. It is intended that while the steps defined by the user may vary, the uncertainties will be the same, and therefore allow documentation about uncertainties to be re-used across projects. This is similar to a threat-based analysis, where uncertainties can threaten the reliability of model output. Uncertainty is preferable as a value-neutral term, as uncertainties are not equally important, and do not pose a threat in all 
situations. As mentioned earlier, focussing on uncertainty also provides a clear link to the concept of risk for decision makers, and to quality assurance as boundary judgement critique. The uncertainty management actions required to be documented for each uncertainty act as process-focussed criteria for the evaluation of each boundary judgement. These criteria were chosen by consideration of consequences if they are omitted or poorly executed (Guillaume et al., 2011, Guillaume et al., 2010). They are compatible with, but more detailed than, the generic actions defined for risk management frameworks (ISO, 2009).

None of the existing approaches gives uncertainty such a central role. The MoST tool gives uncertainty more importance than previously (Refsgaard et al., 2005), requiring predictions to include uncertainty assessments (Refsgaard and Henriksen, 2004). One approach provides model validation tools to address uncertainty (Scholten and Udink ten Cate, 1999). The potential for a documentation process to assist in identifying uncertainties is recognised (Henriksen et al., 2009). Other papers have also alluded to the principles behind this approach. Wood and Mason (1979) talk about "points of corruption". Brade (2000) focus on the "risk [...] that the simulation does not fulfil its intended purpose". Scholten et al. (2001) recognise that "the major risks of modelling are related to the many choices that have to be made. By using its framework focussed on uncertainty, this tool brings all these points together.

\section{DISCUSSION}

Key problems with documentation relate to the substantial effort required to produce it, and the potential difficulties in using it. This problem can be alleviated thanks to advances in technology, consideration of the depth of documentation necessary and considering the documentation production processes. By addressing these problems, the tool hopes to aid adoption.

Advances in technology have helped alleviate problems with distribution of documentation, building of knowledge databases and facilitating querying of the information, far beyond the "vinyl-disk manuals" which were one time seen as the solution (Rosenberg, 1982). These are no longer considered problems in both professional settings, such as software engineering, and everyday life. However, use of software to aid model documentation is still rare (Refsgaard et al., 2005). The prototype for this tool can very easily extract just the information relevant to a particular task, and provides summary reports, enabling for example a hierarchical presentation of results for different audiences (Brade, 2000).

Limiting the detail of documentation simplifies the task. This problem parallels the problem of simplicity vs. complexity in modelling. Documentation should be as concise as possible but no shorter (Rosenberg, 1982, Refsgaard et al., 2005, Wood, 1986). The documentation required here is minimalist - dot point answers have been used in practice, with its meaning enhanced by the structure used. This is similar in intent to classification systems (Highland, 1977) - natural language documentation is not always required. As suggested in the use cases, it is possible to use the documentation approach without completing all details, implying that they are not relevant in a particular case. This is similar in concept to a hierarchy of documentation (Gass et al., 1981). The documentation is simple, but can theoretically still meet common quality characteristics, including being readable, targeted, complete, concise, having a glossary and combined index, being structured, timely, updated, field-tested, and allow feedback (Rosenberg, 1982).

The process used to produce documentation plays an important role, beyond the capabilities offered by software. Clearly defined responsibilities can spread the workload over participants (Henriksen et al., 2009). This tool is recommended to be used for gradual, step-wise reflection, which spreads the burden over time. These options are worth considering, as despite the technology and simplicity, our use indicated that in a single sitting adding detail to existing steps, concentration is difficult to maintain for enough time.

There are evidently other societal issues in the adoption of such tools, such as insufficient funding and time. There are situations where everybody approves of the tool, but nobody uses it. This approach and tool is offered as an aid, without expectation of adoption. It is designed to be easily integrated with existing practices, with a variety of modes of use. This is an advantage over less flexible task-based methods, e.g. for use with stakeholder engagement processes (Henriksen et al., 2009). In contrast with tools that depend on guidelines being accepted, the effectiveness of the tool is not dependent on its widespread acceptance. The tool facilitates review, but does not attempt to guarantee quality. If use of this kind of tool was mandated, it is important to consider the potential for abuse. For guideline-based approaches, there is a risk of following the letter rather than the spirit of the rules. As an example for this tool - if there were pressure to justify choices documented in the tool, it may invite poor responses, jeopardising its usefulness. For this case, it would be recommended that modellers' expertise be embraced - that choices based on the modeller's intuition are acceptable, unless documented evidence exists to refute their position. Evidence that supports the modeller's intuition is not essential. 


\section{CONCLUSION}

A documentation approach and software has been described to enable quality assurance by focussing on uncertainty, but linking to risk. The main features were contrasted with the literature. This tool can play a complementary role to existing approaches, being of particular use where best practice is not established, and a reflective approach is preferable. Issues of effort and adoption of the technique are minimised by the tool design, but how the tool is used in its context will determine its appropriateness and effectiveness.

\section{ACKNOWLEDGEMENTS}

The author would like to acknowledge Tony Jakeman, Barry Croke, Sondoss El Sawah and two anonymous reviewers for their comments on the documentation approach, the software and this paper.

\section{REFERENCES}

Brade, D. (2000) Enhancing modeling and simulation accreditation by structuring verification and validation results, Proceedings of the 32nd Winter Simulation Conference. Orlando, FL.

Checkland, P. \& Holwell, S. (2007) Action Research: Its Nature and Validity, in Kock, N. (Ed.) Information Systems Action Research. Springer US.

Gass, S. I., Hoffman, K. L., Jackson, R. H. F., Joel, L. S. \& Saunders, P. B. (1981) Documentation for a model: a hierarchical approach. Commun. $A C M, 24,728-733$.

Guillaume, J. H. A., Croke, B. F. W., El Sawah, S. \& Jakeman, A. J. (2011) Implementing a framework for managing uncertainty holistically. Watermatex2011. San Sebastian, Spain.

Guillaume, J. H. A. \& Pierce, S. A. (2011) Groundwater Management: What methods have we used to address uncertainty when making decisions? Modflow \& More 2011. Golden, Colorado.

Guillaume, J. H. A., Pierce, S. A. \& Jakeman, A. J. (2010) Managing uncertainty in determining sustainable aquifer yield. Groundwater 2010. Canberra, Australia.

Henriksen, H., Refsgaard, J., Højberg, A., Ferrand, N., Gijsbers, P. \& Scholten, H. (2009) Harmonised Principles for Public Participation in Quality Assurance of Integrated Water Resources Modelling. Water Resources Management, 23, 2539-2554.

Highland, H. J. (1977) A taxonomy approach to simulation model documentation, Proceedings of the 9th Winter simulation Conference, Gaithersburg, MD.

ISO (2009) Risk Management-Principles and Guidelines, ISO 31000:2009, ISO, accessed 1/12/2010.

Jakeman, A. J., Letcher, R. A. \& Norton, J. P. (2006) Ten iterative steps in development and evaluation of environmental models. Environmental Modelling \& Software, 21, 602-614.

Lxml developers. (2011). lxml - XML and HTML with Python. 24/2/2011, http://lxml.de.

Midgley, G. (2000) Systemic intervention: Philosophy, methodology, and practice, Springer US.

Refsgaard, J. C. \& Henriksen, H. J. (2004) Modelling guidelines--terminology and guiding principles. Advances in Water Resources, 27, 71-82.

Refsgaard, J. C., Henriksen, H. J., Harrar, W. G., Scholten, H. \& Kassahun, A. (2005) Quality assurance in model based water management - review of existing practice and outline of new approaches. Environmental Modelling \& Software, 20, 1201-1215.

Refsgaard, J. C., Højberg, A. L., Henriksen, H. J., Scholten, H., Kassahun, A., Packman, J. \& Old, G. (2006) Quality Assurance of the modelling process. In: Proceedings of the XXIV Nordic Hydrological Conference, Vingsted, Denmark

Rosenberg, S. (1982) Computer-aided documentation. Proceedings of the June 7-10, 1982, National Computer conference. Houston, Texas, ACM.

Scholten, H. \& Udink ten Cate, A. J. (1999) Quality assessment of the simulation modeling process. Computers and Electronics in Agriculture, 22, 199-208.

Scholten, H., Van Waveren, R. H., Groot, S., Van Geer, F. C., Wösten, J. H. M., Koeze, R. D. \& Noort, J. J. (2001) Improving the quality of model-based decision support: Good modelling practice in water management. IAHS-AISH Publication, 223-230.

Warmink, J. J., Janssen, J. A. E. B., Booij, M. J. \& Krol, M. S. (2010) Identification and classification of uncertainties in the application of environmental models. Environmental Modelling \& Software, 25, 15181527.

Wood, D. O. (1986) MIT model analysis program: what we have learned about policy model review, Proceedings of the 18th conference on Winter simulation. Washington, D.C., United States, ACM.

Wood, D. O. \& Mason, M. J. (1979) Recommendations concerning energy information model documentation, public access, and evaluation, MIT, Energy Laboratory, Cambridge, USA. 